\title{
Computing Lorentz forces generated by gradient coils in an open MRI system
}

\author{
M. A. Brideson ${ }^{1} \quad$ J. Jackson ${ }^{2} \quad$ L. K. Forbes ${ }^{3}$ \\ S. Crozier ${ }^{4}$
}

(Received 1 August 2007; revised 21 January 2008)

\begin{abstract}
Magnetic Resonance Imaging requires the use of gradient coils to perturb the main homogeneous static magnetic field. Sequential perturbations in each of the orthogonal cartesian directions results in the magnetic field becoming spatially encoded. The fast switching of the gradient coils produces large Lorentz forces which act on the coils. These unwanted forces dissipate as acoustic noise at sound pressure levels upward of $130 \mathrm{~dB}$. We present a method for designing open magnetic resonance imaging coils that produce reduced Lorentz forces whilst still producing highly accurate gradient fields. We show that when gradient fields of approximately $1 \%$ are considered, a linearised regularisation solves for the Fourier series coefficients.
\end{abstract}

See http://anziamj.austms.org.au/ojs/index.php/ANZIAMJ/article/view/366 for this article, (C) Austral. Mathematical Soc. 2008. Published January 29, 2008. ISSN 1446-8735 


\section{Contents}

1 Introduction

C424

2 Mathematical model

$\mathrm{C} 426$

2.1 Magnetic fields, streamfunctions, and Lorentz forces . . . .

C429

2.2 Regularisation . . . . . . . . . . . . . .

3 Results

C431

4 Conclusion

C435

References

C436

\section{Introduction}

A Magnetic Resonance Imaging (MRI) device is an apparatus designed for non-invasive imaging of the internal structure of an object. It delivers high spatial resolution, and is highly sensitive to molecular differences. These attributes are ideal in medical applications since they permit high constrast images showing extremely accurate delineation of tissue in the body.

An MRI system is a compound set of magnets and coils. There are four main types of magnets: the main magnet, shim coils, gradient coils, and radiofrequency $(\mathrm{RF})$ coils. The main magnet produces a large static homogeneous magnetic field, typically of the order of 1-10 tesla. For the purposes of imaging, the only component of the magnetic flux density $\boldsymbol{B}$ that we require is its $z$ component, $B_{z}$. Further references to this component of the magnetic flux density will be via the terms 'the magnetic field' or simply 'the field'.

Errors in the static magnetic field are always present, primarily due to engineering and manufacturing limitations. Shim coils are used to correct the inaccuracies. Any shim coil is designed to excite a particular spherical 
harmonic in the decomposed main field. Due to orthogonality, the excitation of any particular shim coil should have no impact on the performance of any other shim coil.

With an extremely accurate static field in place, known perturbations are then superimposed on the main field by a set of gradient coils. The name stems from the field perturbations being linear in $x, y$ and $z$, with gradients in the order of $10-50 \mathrm{mT} / \mathrm{m}$. By the Larmor relationship $\omega=\gamma B$, when the static field $B_{0}$ is perturbed by $\Delta B_{0}$, the precessional angular velocity $\omega$ of any magnetic moment will perturb by $\Delta \omega$ under the same linear relationship:

$$
(\omega+\Delta \omega)=\gamma\left(B_{0}+\Delta B_{0}\right) .
$$

The gyromagnetic ratio $\gamma$ is a constant for any given nucleus, and due to its abundance in the human body, the hydrogen nucleus is targetted for MRI.

The final major set of coils in the MRI system is the set of RF coils. They transmit RF energy into the sample at the Larmor frequency for hydrogen, thus causing excitation of the hydrogen nuclei. When the RF energy is removed, the nuclei return to their previous state by re-emitting the absorbed energy. The time taken for relaxation is linked to the molecular structure of individual tissue and therefore is the source of contrast in images.

Since the field exhibits linear variation due to the gradient coils, so too does the Larmor frequency. Thus, by sequential application of the gradient coils in the three cartesian directions, any volume element in the imaging region can be isolated by way of the applied gradient field. The task of gradient coils is commonly referred to as 'spatial encoding'.

The focus of this report is on the use of gradient coils. In particular, our interest lies in the reduction of acoustic noise levels produced by their switching on and off. When switched on, the field from a gradient coil quickly ramps up to its desired level, remains constant for a short period, and then switches off. As the current density in the coil rapidly changes, and the magnetic field also rapidly changes, significant Lorentz forces act on the 
coil. With the coils fixed in place, these forces are dissipated acoustically at pressure levels of approximately 100-130 decibels [3, 4]. The threshold of painful hearing is at $130 \mathrm{~dB}$, so it is in the interest of the MRI engineer to design quieter coils.

The traditional geometry for an MRI system is a horizontal cylindrical structure with a central bore into which the patient is placed. The coil and magnet system surround the bore. Many patients find this configuration uncomfortable as the confined space and loud noise can lead to severe anxiety and claustrophobia. Some modern MRI designs attempt to overcome these shortcomings with an open cylindrical geometry. In this configuration the magnets are on the cylinder ends and the sides are open. It is this configuration that is assessed in this project.

Section 2.1 derives Fourier series expressions for the $B_{z}$ distribution, stream functions, and Lorentz forces acting on the shielded gradient coils in the presence of the main field and the gradient field. Section 2.2 constructs a regularisation formula that provides a trade-off between accuracy of the magnetic field and magnitude of the Lorentz forces. Section 3 shows that for an unshielded system, when the gradient field is about $1 \%$ of the main field, optimisation of this regularisation formula can be linearised with respect to the Fourier coefficients.

\section{Mathematical model}

In the open coil arrangement, a gradient coil is composed of two biplanar primary coils located at $z= \pm d_{p}$ and external to these, two biplanar shield coils located at $z= \pm d_{s}$ (Figure 1). Each coil is a conducting sheet into which etchings are made to force a particular current density distribution.

In the regions not including the coils, a magnetic scalar potential $\varphi$ exists that satisfies Laplace's equation $\nabla^{2} \varphi=0$. The magnetic flux density $\boldsymbol{B}$ in 


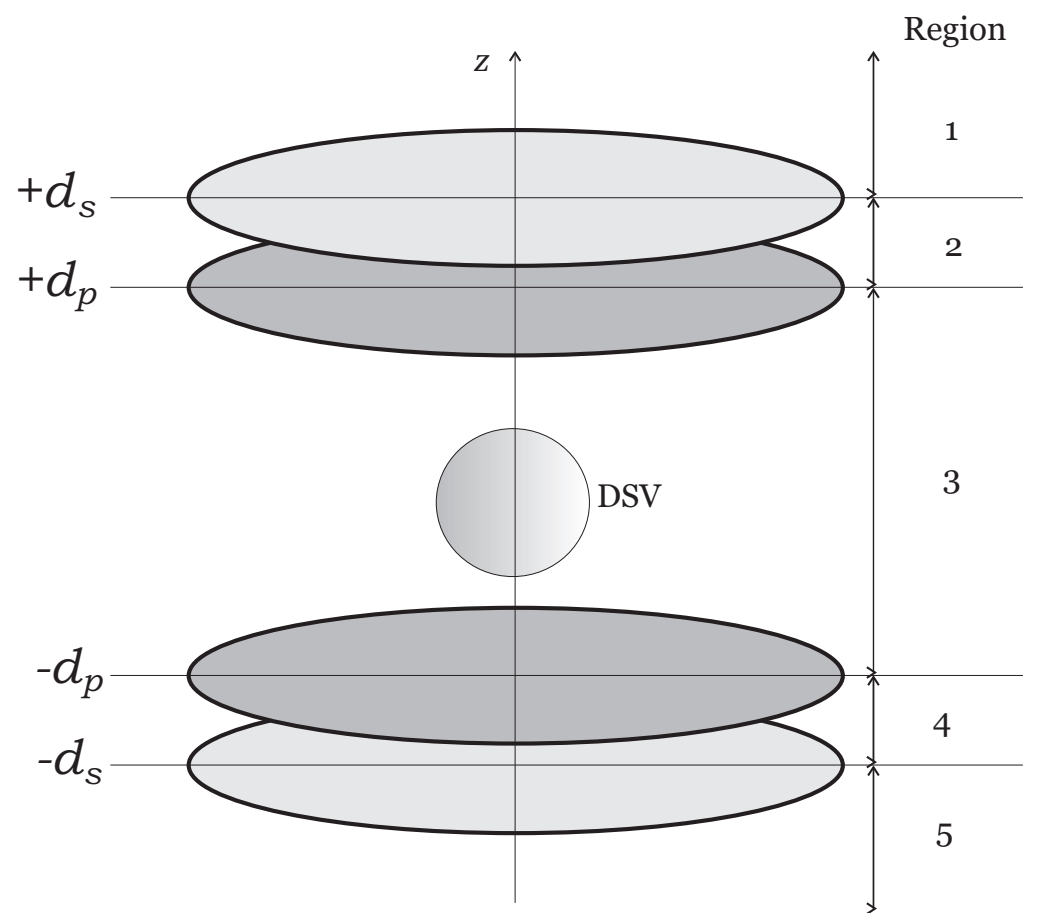

FIGURE 1: A schematic diagram of a shielded system of open MRI coils. 
each of these regions is derived from the gradient of the corresponding scalar potential, $\boldsymbol{B}=-\nabla \varphi$.

In Figure 1, regions 1 and 5 are external to the coil system and ideally the magnetic flux density should be zero here. This is accomplished by the shielding coils actively nullifying the field of the primary coils. However, the shielding coils also contribute to the field of the primary coils in region 3 where imaging is performed. To produce accurate images for medical applications, the field must be known to parts per billion accuracy within a small spherical region in region 3 , known as the diameter sensitive volume (DSV).

In designing the gradient coils, we have used a modified version of the target field method introduced by Turner [1]. In Turner's target field method, a desired magnetic field is defined throughout the DSV and its Fourier transform spectrum is recorded. By way of the inverse Fourier transform, this spectrum is then used to obtain predictions for the magnetic flux density in each of the five regions, and for the current density distributions on each of the four coils. A consequence of using Fourier transforms is that coil lengths are implied to be infinite. Turner proposed apodization as a way to force the current density to zero outside the dimensions of the proposed coil, thus forcing the coils to be finite.

The modified approach adopted for this project is to use Fourier-Bessel series expansions rather than Fourier transform specifications for all vector fields. In this way the finite dimensions of the coil are treated automatically without the need to artificially force the coil dimensions. In addition, the winding patterns on the coils are plotted using stream functions related to the current density as outlined by Brideson [2]. Also, the Lorentz forces are obtained from the cross product operation involving the Fourier series expressions for current density and magnetic field. 


\subsection{Magnetic fields, streamfunctions, and Lorentz forces}

The cartesian gradients are written in cylindrical polar form where $x=$ $r \cos \phi, y=r \sin \phi$ and $z$ remains unchanged. Solving Maxwell's equations in static form with appropriate boundary conditions on the coils leads to the following specifications for the $k$ th general term of $B_{z}$ in the imaging region for an $x$ gradient,

$$
B_{z}(r, \phi, z)=C_{1}^{p+} k \cosh (k z)\left(\frac{e^{2 k d_{p}}-e^{2 k d_{s}}}{1+e^{2 k d_{s}}}\right) J_{1}(k r) \cos \phi ;
$$

and for a $y$ gradient,

$$
B_{z}(r, \phi, z)=C_{1}^{p+} k \cosh (k z)\left(\frac{e^{2 k d_{p}}-e^{2 k d_{s}}}{1+e^{2 k d_{s}}}\right) J_{1}(k r) \sin \phi .
$$

These equations are compared against the target field $B_{z}^{T}(r, \phi ; z= \pm c)$ for an $x$ gradient,

$$
B_{z}^{T}(r, \phi ; z= \pm c)=a_{k} J_{1}(k r) \cos \phi ;
$$

and $y$ gradient,

$$
B_{z}^{T}(r, \phi ; z= \pm c)=a_{k} J_{1}(k r) \sin \phi .
$$

Note that the target field is defined on the DSV which is now considered to be a cylinder of height $2 c$. By equating equations (1) and (3), and equations (2) and (4) respectively, $B_{z}$ is rewritten in terms of the Fourier coefficients $a_{k}$ of the target field. For the $x$ and $y$ gradients, the respective $B_{z}$ fields are

$$
\begin{aligned}
& B_{z}(r, \phi, z)=a_{k} J_{1}(k r) \cos \phi \frac{\cosh (k z)}{\cosh (k c)}, \\
& B_{z}(r, \phi, z)=a_{k} J_{1}(k r) \sin \phi \frac{\cosh (k z)}{\cosh (k c)} .
\end{aligned}
$$

The specification for the $z$ gradient is similar, but with the $\phi$ dependence removed, cosh replaced with sinh, and the first order Bessel functions now 
zeroth order:

$$
B_{z}(r, \phi, z)=a_{k} J_{0}(k r) \frac{\sinh (k z)}{\sinh (k c)}
$$

The winding patterns on each of the coils are given by contours of the streamfunction $\psi(r, \phi, z)$, which is derived from the current density $\boldsymbol{j}$ by way of the continuity equation:

$$
\nabla \cdot \boldsymbol{j}=\nabla \cdot\left(\nabla \times \psi \mathbf{e}_{\mathbf{z}}\right)=0 .
$$

For the $x$ and $y$ gradients, the winding patterns on the two primary coils are identical,

$$
\psi_{p}\left(r, \phi ; z= \pm d_{p}\right)=-a_{k} \frac{2 J_{1}(k r) e^{k d_{p}}}{\mu_{0} k \cosh (k c)}\left(\frac{1+e^{2 k d_{s}}}{e^{2 k d_{p}}-e^{2 k d_{s}}}\right),
$$

as are the winding patterns on the two shields,

$$
\psi_{s}\left(r, \phi ; z= \pm d_{s}\right)=a_{k} \frac{2 J_{1}(k r) e^{k d_{s}}}{\mu_{0} k \cosh (k c)}\left(\frac{1+e^{2 k d_{p}}}{e^{2 k d_{p}}-e^{2 k d_{s}}}\right) .
$$

Equations (9) and (10) are multiplied by $\cos \phi$ for an $x$ gradient and $\sin \phi$ for a $y$ gradient, with $\mu_{0}$ the permeability of free space.

For a $z$ gradient, the winding patterns on the two primary coils are opposite in their orientation,

$$
\psi_{p}\left(r, \phi ; z= \pm d_{p}\right)=\mp a_{k} \frac{2 J_{0}(k r) e^{k d_{p}}}{\mu_{0} k \sinh (k c)}\left(\frac{e^{2 k d_{s}}-1}{e^{2 k d_{p}}-e^{2 k d_{s}}}\right),
$$

as are the winding patterns on the two shields,

$$
\psi_{s}\left(r, \phi ; z= \pm d_{s}\right)= \pm a_{k} \frac{2 J_{0}(k r) e^{k d_{s}}}{\mu_{0} k \sinh (k c)}\left(\frac{e^{2 k d_{p}}-1}{e^{2 k d_{p}}-e^{2 k d_{s}}}\right) .
$$

Since no current flows perpendicular to the coils, the Lorentz force is computed from the current density and magnetic field by

$$
\begin{aligned}
\boldsymbol{F} & =\boldsymbol{j} \times \boldsymbol{B} \\
& =j_{\phi}\left(B_{0}+B_{z}\right) \hat{\boldsymbol{e}}_{r}-j_{r}\left(B_{0}+B_{z}\right) \hat{\boldsymbol{e}}_{\phi}+\left(j_{r} B_{\phi}-j_{\phi} B_{r}\right) \hat{\boldsymbol{e}}_{z} .
\end{aligned}
$$




\subsection{Regularisation}

To reduce the impact of Lorentz forces, we begin by constructing a regularisation formula $R$ that includes a Lorentz force term, a term matching the magnetic field to the target field in the DSV, and a term to null the magnetic field external to the coil system at $|z|>d_{s}$ :

$$
\begin{aligned}
R= & \iint_{\mathrm{DSV}}\left[B_{z}^{T}(r, \phi, c)-B_{z}(r, \phi, c)\right]^{2} d S \\
& +\delta_{s} \iint_{\text {regions } 1,5}\left[B_{z}\left(r, \phi,|z|>d_{s}\right)-0\right]^{2} d S \\
& +\lambda \iint_{\text {coils }}\|\boldsymbol{F}\|^{2} d S .
\end{aligned}
$$

An unshielded system is considered by setting $\delta_{s}=0$; shielding is included with $\delta_{s}=1$. When shielding is ignored, the external regions become regions 1 and 2 and regions 4 and 5 .

With the regularisation parameter $\lambda$ set to zero, and equation (14) minimised with respect to the Fourier coefficients $\left(\partial R / \partial a_{k}=0\right)$, the standard Euler-type formulae result for the Fourier-Bessel coefficients. As $\lambda$ increases away from zero, the effect of the force term is increased at the expense of accuracy in the magnetic fields. Choosing the optimum value for $\lambda$ thus comes down to a subjective decision on the relative importance of field accuracy over force reduction.

\section{Results}

The non-dimensionalised coil configuration under test has all coils of negligible thickness, radius $b=1.0$, and positioned such that $d_{p}=1$ and $d_{s}=1.1$. To produce a $z$ gradient the target field must be constant in $x$ and $y$, with a 
linear variation between the target depths, $z= \pm c$. Due to convergence issues associated with the Fourier coefficients, the target depths must be set to $c= \pm d_{p}$. Hence the target field is defined such that $B_{z}^{T}\left(R<b, \phi, \pm d_{p}\right)= \pm 1$ and on the boundaries of the coils, $B_{z}^{T}\left(b, \phi, \pm d_{p}\right)=0$.

For the purpose of illustration we considered three coil systems:

1. a shielded and unregularised system $\left(\delta_{s}=1, \lambda=0\right)$;

2. an unshielded and unregularised system $\left(\delta_{s}=0, \lambda=0\right)$; and

3. an unshielded and regularised system $\left(\delta_{s}=0, \lambda=0.05\right)$ - this value of $\lambda$ was chosen purely for illustrative purposes.

An analysis of the Lorentz forces for the shielded and unregularised system shows that we can ignore $F_{z}$ in equation (13) if the gradient perturbs the main field by a small amount. Figure 2 shows this where the $r$ and $z$ components of the force on the primary and shield coils are shown for $z$ gradients producing maximal perturbations in the main field of $1 \%$ and $5 \%$. Additionally, $F_{\phi}$ is zero since no radial current component exists for a $z$ gradient.

In both the unshielded cases, we chose $B_{z} \ll B_{0}$ so that $\|\boldsymbol{F}\| \approx F_{r} \approx$ $j_{\phi} B_{0}$. This has the significant benefit of making the regularisation process linear in $a_{k}$ rather than cubic.

Figure 3 gives profiles of $B_{z}$ across the five regions of interest for the three coil systems. In the central region, wherein the DSV is located, the unregularised systems produce field distributions (orange and green lines) close to the linear target field (dashed line) for smaller values of $r$. The mismatch exhibits a sinh variation as expected from equation (7), that amplifies as the radius increases. From an imaging point of view, this falloff is not deleterious as it is only within the small DSV region that the actual and ideal fields must be well matched. 

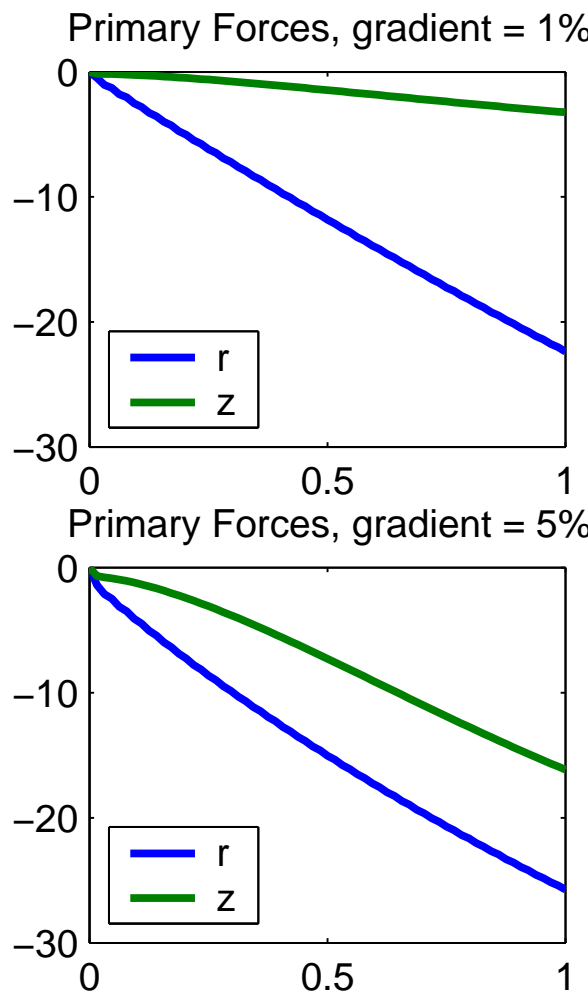

Shield Forces, gradient $=1 \%$

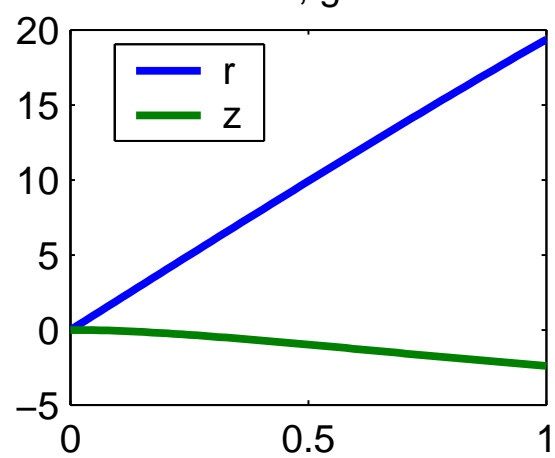

Shield Forces, gradient $=5 \%$

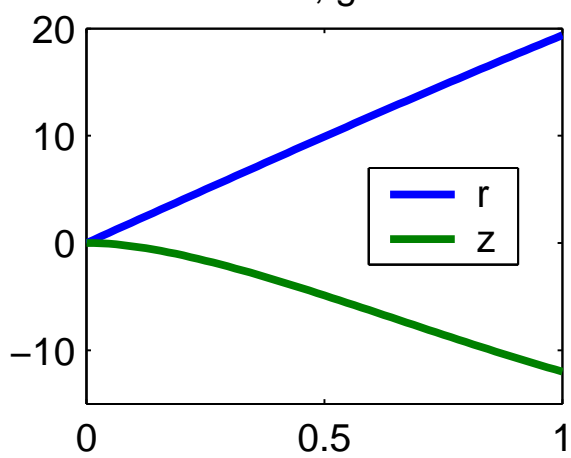

FIGURE 2: $r$ and $z$ components of the Lorentz force on the primary and shield coils for $1 \%$ and $5 \% z$ gradients in the shielded and unregularised system. 

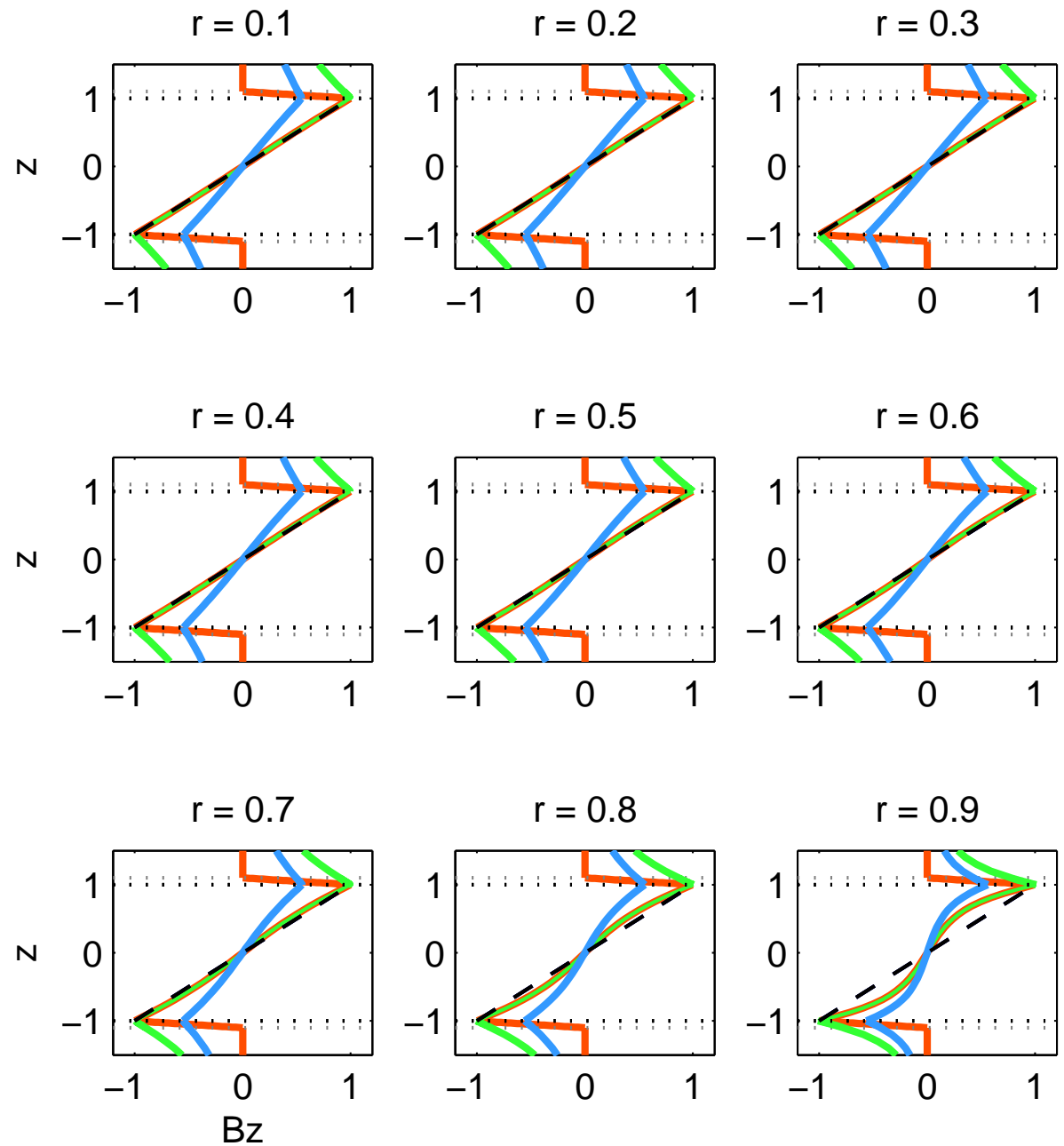

FIGURE 3: Fourier series computations of $B_{z}$ at varying cylindrical radii $r$ across the five regions for a shielded and unregularised setup (orange line), an unshielded and unregularised setup (green line), and an unshielded and regularised setup (blue line). The dotted lines indicate the positions of the primary coils (black) and shield coils (grey), and the dashed line indicates the target field. 

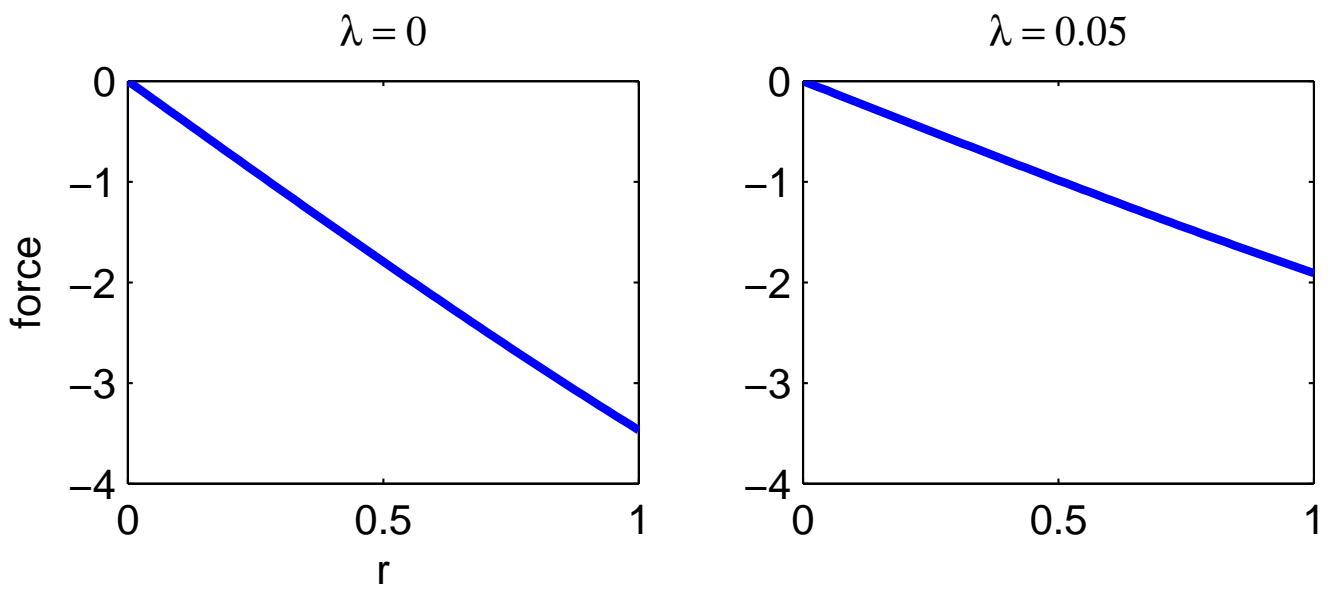

FiguRE 4: $r$ component of the Lorentz force on the primary coil for a $1 \% z$ gradient in an unshielded and unregularised system $(\lambda=0)$ and an unshielded but regularised system $(\lambda=0.05)$.

For an unshielded system with nonzero regularisation parameter the mismatch is more pronounced. This is also to be expected as field accuracy has been traded for a decrease in Lorentz forces, Figure 4. For a $z$ gradient the coil windings are circularly symmetric like the well known Maxwell coil gradient system. Since the coil dimensions and separations are fixed, only the radius of the windings and the magnitude of the current can produce the reduction in force. In a fully regularised system, the ability to change the dimensions and relative locations of the coils might also be considered.

\section{Conclusion}

We have presented a method based on Fourier-Bessel series for computing the magnetic fields and Lorentz forces generated by a shielded set of gradient 
coils in an open configuration. In the next stage of this project we will minimise the regularisation formula, equation (14), for the full shielded system, exploring the impact of the regularisation parameter $\lambda$. We will also adapt our technique to permit multiple target depths, rather than just the primary coil depth which is forced upon us by convergence issues. This should provide a better match between the target field and computed field [5]. We also plan to extend the model to incorporate minimisation of coil deflections and sound pressure levels, both of which are consequentially related to Lorentz forces [6].

\section{References}

[1] R. Turner. A target field approach to optimal coil design. J. Phys. D: Appl. Phys., 19, 1986, pages L147-L151. doi:10.1088/0022-3727/19/8/001 C428

[2] M. A. Brideson, L. K. Forbes and S. Crozier. Determining complicated winding patterns for shim coils using stream functions and the target-field method. Concepts in Magnetic Resonance, 14(1), 2002, pages 9-18. doi:10.1002/cmr.10000 C428

[3] P. Mansfield, P. M. Glover and J. Beaumont. Sound generation in gradient coil structures for MRI. Magnetic Resonance in Medicine, 39(4), 1998, 539-550. doi:10.1002/mrm.1910390406 C426

[4] P. Mansfield, B. Haywood and R. Coxon. Active Acoustic Control in Gradient Coils for MRI. Magnetic Resonance in Medicine, 46(4), 2001, 807-818. doi:10.1002/mrm.1261 C426

[5] M. A. Brideson, L. K. Forbes and S. Crozier. Winding patterns for actively shielded shim coils with asymmetric target-fields. Measurement Science and Technlogy, 14, 2003, pages 484-493. doi:10.1088/0957-0233/14/4/312 C436 
[6] L. K. Forbes, M. A. Brideson, S. Crozier and P. T. While, An analytical approach to the design of quiet cylindrical asymmetric gradient coils in MRI. Concepts in Magnetic Resonance Part B. 31B(4), 2007, 218-236. doi:10.1002/cmr.b.20095. C436 


\section{Author addresses}

1. M. A. Brideson, School of Mathematicss \& Physics, University of Tasmania, Hobart, Australia.

mailto:michael.brideson@utas . edu . au

2. J. Jackson, School of Mathematicss \& Physics, University of Tasmania, Hobart, Australia.

3. L. K. Forbes, School of Mathematicss \& Physics, University of Tasmania, Hobart, Australia.

4. S. Crozier, School of Information Technology \& Electrical Engineering, The University of Queensland, Australia. 